\title{
Novel de novo FOXC1 nonsense mutation in an Axenfeld- Rieger syndrome patient
}

\author{
Susana Carmona ${ }^{1,2}$ (D) | Maria da Luz Freitas ${ }^{3}$ | Hugo Froufe $^{1}$ | \\ Maria José Simões $^{1}$ | Maria João Sampaio ${ }^{4}$ | Eduardo D. Silva ${ }^{5}$ | \\ Conceição Egas ${ }^{1,6}$
}

${ }^{1}$ Next-Gen Sequencing Unit, UC-Biotech, Cantanhede, Portugal

${ }^{2}$ Faculty of Medicine, University of Coimbra, Coimbra, Portugal

${ }^{3}$ Department of Ophthalmology, Hospital da Luz-Arrábida, Porto, Portugal

${ }^{4}$ Department of Paediatrics, Hospital CUF Porto, Porto, Portugal

${ }^{5}$ Faculty of Medicine, IBILI, University of Coimbra, Coimbra, Portugal

${ }^{6}$ Center for Neuroscience and Cell Biology, University of Coimbra, Coimbra, Portugal

Correspondence

Conceição Egas, UC-Biotech, Biocant-Park, Núcleo 04, Lote 8, Cantanhede 3060-197, Portugal.

Email: cegas@biocant.pt

Funding information

Fundação para a Ciência e a Tecnologia, Grant number: SFRH/BD/90445/2012; Programa Operacional Potencial Humano/Fundo Social Europeu (POPH/FSE)

To the Editor:

Axenfeld-Rieger Syndrome (ARS) (OMIM 602482) is a rare developmental disorder characterized by anterior segment dysgenesis, affecting the peripheral cornea and iris, and iridocorneal angle. Iris attachments to the posterior embryotoxon (prominent Schwalbe's line) in both eyes characterize ARS patients. Other iris findings might include stromal thinning or atrophy, corectopia, iris holes, and ectropion uvea. A major consequence of the anterior segment dysgenesis is increased intraocular pressure, leading to glaucoma, in approximately half of the ARS patients. Extraocular findings in ARS are also present and may include craniofacial anomalies such as maxillary and occasionally mandibular hypoplasia, hypertelorism, telecanthus, micro-hypodontia, cardiovascular malformations, anomalies of the pituitary gland, growth hormone deficiency, genitourinary anomalies like hypospadias in males, or periumbilical skin redundancy (Chang, Summers, Schimmenti, \& Grajewski, 2012; Tumer \& BachHolm, 2009).

Other disorders may have phenotypic overlapping with ARS, such as hypoplasia/iridogoniodysgenesis anomaly/syndrome, Peters anomaly, and infantile/primary congenital glaucoma (Tumer \& Bach-Holm, 2009). The most important disorder to consider is Peters anomaly, an iridogoniodysgenesis, usually bilateral, appearing in the same age group. Patients with this disease may also present posterior embryotoxon, iris coloboma, aniridia, and glaucoma, as in ARS patients. However, the disease is characterized by the presence of one or more central corneal opacities caused by central absence of the corneal endothelium, Descemet's membrane and posterior corneal stroma. The central corneal opacities are absent in ARS (Weisschuh, Wolf, Wissinger, \& Gramer, 2008).

ARS is an autosomal dominant disorder mainly affecting eye with a genetic heterogeneity and estimated prevalence of 1 in 200,000. It is associated with mutations and copy number changes in two transcription factor genes: PITX2 (Semina et al., 1996), and FOXC1 (Mears et al., 1998). PITX2 is mutated more commonly in patients with dental and/or umbilical anomalies while FOXC1 mutations are revealed more frequently in cases with isolated ocular findings or in association with heart and/or hearing defects (Reis et al., 2012). Sporadic cases have also been reported with CYP1B1 (Tanwar, Dada, \& Dada, 2010), and PRDM5 mutations (Micheal et al., 2016).

In this study, we report on a 14-month-old Portuguese male presenting with typical features of ARS (Table 1, Figure 1), including bilateral glaucoma. No anterior segment anomalies were present in the parents. Hypertelorism, maxillary hypoplasia, dental anomalies, and short stature (below 3rd centiles [World Health Organization, 2009]) led to the preliminary diagnosis of ARS. The boy also had frontal prominence, broad nasal root, and unilateral testicular atrophy. Neither cardiac malformations, also confirmed by normal echocardiography, nor redundant periumbilical skin were present. At 23 months of age, growth hormone, and IGF1 levels were in normal limits and his growth parameters and neuromotor development were reported as normal. 
TABLE 1 Biometric parameters revealed by detailed opthalmological examination

\begin{tabular}{lll}
\hline Parameters & Right eye & Left eye \\
\hline Horizontal corneal diameter $(\mathrm{mm})$ & 14 & 13 \\
\hline Vertical corneal diameter $(\mathrm{mm})$ & 13.5 & 13 \\
\hline Axial length $(\mathrm{mm})$ & 21.61 & 21.26 \\
\hline Anterior chamber $(\mathrm{mm})$ & 3.96 & 3.67 \\
\hline IOP $(\mathrm{mmHg}$, by Perkins tonometer, under anesthesia, and maleate timolol) & 20 & 18 \\
\hline US central corneal thickness & 656 & 612 \\
\hline Haab striae & Yes & No \\
\hline Posterior embryotoxon & Yes & Yes \\
\hline Iridocorneal extensions & Yes & Yes \\
\hline Cup/disc & Large disc and large cup & Large disc and large cup \\
\hline
\end{tabular}

IOP, intraocular pressure; US, ultrasound.

Note that posterior embryotoxon, iridocorneal extensions were present in both eyes. Corneal diameter, central corneal thickness, anterior chamber, and axial length were increased in both eyes. High intraocular pressure was noted bilaterally while Haab striae was observed only in the right eye. Normative values for the biometric parameters are in supplementary material S1.

The family provided informed consent for research and publication in act to Helsinki Declaration. Whole-exome sequencing was performed on genomic DNA of the proband using lon AmpliSeq ${ }^{\mathrm{TM}}$ RDY Exome Kit with the lon Proton System (Life Technologies). After the run, the sequences were aligned to the hg19/GRCh37 reference genome and the variants annotated by ANNOVAR. SNPs and Indels were filtered to identify the disease-causing variant and the related gene. Exonic and splice-site variants were selected for minor allele frequency (MAF) inferior to $1 \%$, according to the information in the European population of the 1,000 Genome Project, NHLBI Exome Sequencing Project and an in-house database. Functional impact of the remaining variants was evaluated based on the scores provided by SIFT, PolyPhen-2, MutationTaster, and CADD software. Inter-species conservation scores were provided by PhyloP and GERP++ software.

The genes related with ARS phenotype, FOXC1, PIXT2, CYP1B1, and PRDM5, were considered as possible candidate genes and analysed to identify causal pathogenic variants. The patient presented the heterozygous nonsense variant p.Tyr64Ter (c.192 C > G) in FOXC1 (Figure 2). No other variants were identified in the $3^{\prime} U T R$ of this gene or in the coding regions of PITX2, CYP1B1, and PRDM5. The FOXC1 variant, c.192 C > G, was further confirmed by Sanger sequencing in the proband and excluded in the parents revealing de novo state for autosomal dominant inheritance pattern. The mutation was not observed in public databases neither in a Portuguese control population.

The in silico functional impact evaluated by MutationTaster classified the variant as "disease causing" and the CADD software indicated a high scaled score of 36 , suggesting the deleterious effect of the mutation. This p.Tyr64Ter mutation is located before the DNA-binding forkhead domain (FHD) (Figure 2c), resulting in a truncated protein composed only of the activation domain with loss of $88.6 \%$ of the protein. Consequently, the FHD, the inhibition
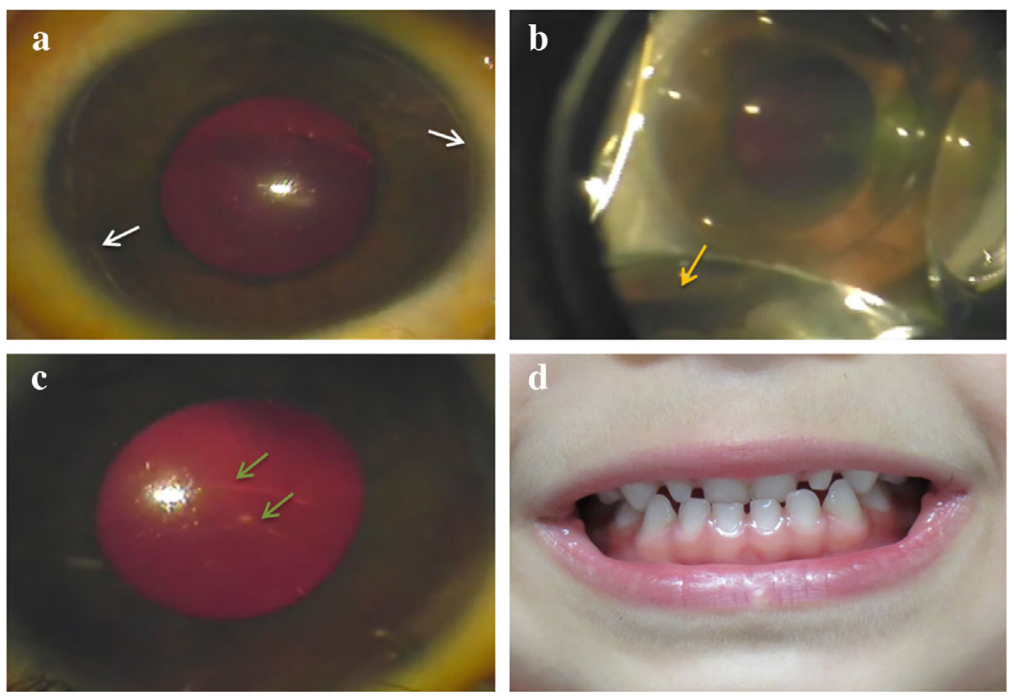

FIGURE 1 Ocular and dental changes observed in the proband. (a) Embryotoxon (white arrows). (b) Iridocorneal angle showing iridocorneal extensions (yellow arrow). (c) Haab Striae (green arrows). (d) Microdontia. [Color figure can be viewed at wileyonlinelibrary.com] 

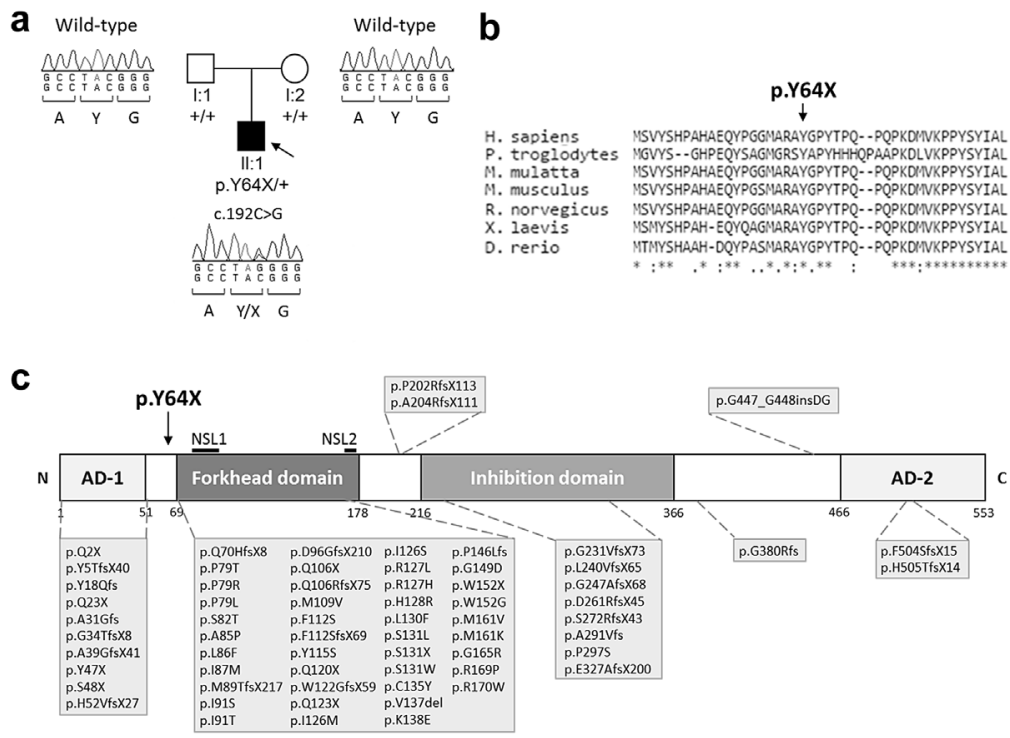

FIGURE 2 (a) Pedigree of the family and Sanger sequencing electropherograms showing c.192 C> G (p.Tyr64Ter) FOXC1 mutation at heterozygote state in the proband and wild-type allele in both parents. (b) Multiple amino acid alignment of the FOXC1 protein of known vertebrates. The novel mutation p.Tyr64Ter occurs in a highly conserved amino acid across the species. Alignments were performed with Clustal Omega. (c) Localization of the p.Tyr64Ter FOXC1 mutation before the forkhead domain and overview of FOXC1 mutations. AD-1: activation domain 1; AD-2: activation domain 2; NSL1: nuclear localization signal 1; NSL2: nuclear localization signal 2

domain, and the activation domain two will be absent. In a recent study, Medina-Trillo and collaborators described an increased transactivation activity in two nonsense mutations: p.Tyr47Ter and p.GIn106Ter. Similar to the mutation revealed in our patient, p.Tyr47Ter also resides before the FHD. The authors suggested that this unexpected gain-of-function might be related to the isolated expression of the activation domain 1, without the inhibitor domain. The activation domain 1 appears to act as a potent and independent transcription up-regulator that can interact with other transcription factors beyond FOXC1 (Medina-Trillo et al., 2015). According to this data, we hypothesize that the de novo mutation identified in our study can create a hypermorphic allele, being responsible for the ARS phenotype observed in the patient.

The mechanism behind the development of glaucoma caused by FOXC1 disruption is not clearly understood. This transcription factor is expressed during eye organogenesis in the periocular mesenchyme, trabecular meshwork, sclera, and conjunctival epithelium (Kidson, Kume, Deng, Winfrey, \& Hogan, 1999). It has a significant role in the normal corneal development, preserving corneal transparency by regulating vascular growth, and is also involved in the correct iris and trabecular meshwork formation (Kidson et al., 1999; Seo et al., 2012). Studies performed in human trabecular meshwork (TM) cells revealed the important role of FOXC1 in the response to oxidative stress and FOXC1 dysregulation results in TM cell death. The loss of TM cellularity will prevent aqueous humor uptake with a consequent increase in intraocular pressure (IOP), a major risk factor for the development of glaucoma (Berry et al., 2008; Ito, Goping, Berry, \& Walter, 2014).

In addition to the candidate approach, all exonic and splice site variants, excluding synonymous variants, were evaluated for pathogenicity according to ClinVar database classification or for association with a disease on the GWAS catalog. The purpose of this analysis was to find possible associations between the other variants and the patient's features. A common previously described variant in COL11A1 identified by GWAS as a susceptibility locus for primary angle closure glaucoma (PACG) (Vithana et al., 2012) was also found at heterozygote state in the patient: p.Pro1323Leu (c.3968C > T, rs3753841). COL11A1 encodes the alpha chain of collagen type $\mathrm{XI}$ and pathogenic mutations in this gene are causative of syndromes with ocular involvement. This protein is a component of the extracellular matrix of human TM and, therefore, contributes to its structure (Acott \& Kelley, 2008; Vithana et al., 2012). Despite rs3753841 being predicted to have a moderate effect on the protein, a small aberrant activity or expression, in the presence of the hypermorphic FOXC1 allele, could contribute to a disruption of the TM and aqueous humor drainage with IOP increase. Further studies will clarify the possible impact of this variant in association with FOXC1 in the pathogenesis of glaucoma in ARS patients.

In conclusion, molecular screening by WES for ARS which has genetic heterogeneity has led to the identification of a novel nonsense mutation in FOXC1. This variant expanded the spectrum of FOXC1 mutations associated with ARS. WES leads to fast, easy screening of several genes simultaneously to identify genetic etiopathogenesis and in cases where surveillance and treatment strategies are based on molecular data could be considered as the first tier testing. Furthermore, WES analysis may provide additional data on accompanying variants which may also have an impact on the clinical phenotype.

\section{ACKNOWLEDGMENTS}

We thank the family members for their participation. Susana Carmona was supported by a doctoral fellowship from Fundação 
para a Ciência e a Tecnologia (SFRH/BD/9044/2012), Programa Operacional Potencial Humano/Fundo Social Europeu (POPH/FSE).

\section{CONFLICTS OF INTEREST}

The authors report no competing, commercial, or conflicts of interests. The authors are responsible for the content and writing of the paper.

\section{REFERENCES}

Acott, T. S., \& Kelley, M. J. (2008). Extracellular matrix in the trabecular meshwork. Experimental Eye Research, 86(4), 543-561.

Berry, F. B., Skarie, J. M., Mirzayans, F., Fortin, Y., Hudson, T. J., Raymond, V., ... Walter, M. A. (2008). FOXC1 is required for cell viability and resistance to oxidative stress in the eye through the transcriptional regulation of FOXO1A. Human Molecular Genetics, 17(4), 490-505.

Chang, T. C., Summers, C. G., Schimmenti, L. A., \& Grajewski, A. L. (2012). Axenfeld-Rieger syndrome: New perspectives. British Journal of Ophthalmology, 96(3), 318-322.

Ito, Y. A., Goping, I. S., Berry, F., \& Walter, M. A. (2014). Dysfunction of the stress-responsive FOXC1 transcription factor contributes to the earlier-onset glaucoma observed in Axenfeld-Rieger syndrome patients. Cell Death \& Disease, 5, e1069.

Kidson, S. H., Kume, T., Deng, K., Winfrey, V., \& Hogan, B. L. (1999). The forkhead/winged-helix gene, Mf1, is necessary for the normal development of the cornea and formation of the anterior chamber in the mouse eye. Developmental Biology, 211(2), 306-322.

Mears, A. J., Jordan, T., Mirzayans, F., Dubois, S., Kume, T., Parlee, M., .. Walter, M. A. (1998). Mutations of the forkhead/winged-helix gene, FKHL7, in patients with Axenfeld-Rieger anomaly. American Journal of Human Genetics, 63(5), 1316-1328.

Medina-Trillo, C., Sanchez-Sanchez, F., Aroca-Aguilar, J. D., Ferre-Fernandez, J. J., Morales, L., Mendez-Hernandez, C. D., ... Escribano, J. (2015). Hypo- and hypermorphic FOXC1 mutations in dominant glaucoma: Transactivation and phenotypic variability. PLoS ONE, 10(3), e0119272.

Micheal, S., Siddiqui, S. N., Zafar, S. N., Venselaar, H., Qamar, R., Khan, M. I., $\&$ den Hollander, A. I. (2016). Whole exome sequencing identifies a heterozygous missense variant in the PRDM5 gene in a family with Axenfeld-Rieger syndrome. Neurogenetics, 17(1), 17-23.

Reis, L. M., Tyler, R. C., Volkmann Kloss, B. A., Schilter, K. F., Levin, A. V., Lowry, R. B., ... Semina, E. V. (2012). PITX2 and FOXC1 spectrum of mutations in ocular syndromes. European Journal of Human Genetics, 20(12), 1224-1233.

Semina, E. V., Reiter, R., Leysens, N. J., Alward, W. L., Small, K. W., Datson, N. A., ... Murray, J. C. (1996). Cloning and characterization of a novel bicoid-related homeobox transcription factor gene, RIEG, involved in Rieger syndrome. Nature Genetics, 14(4), 392-399.

Seo, S., Singh, H. P., Lacal, P. M., Sasman, A., Fatima, A., Liu, T., ... Kume, T. (2012). Forkhead box transcription factor FoxC1 preserves corneal transparency by regulating vascular growth. Proceedings of the National Academy of Sciences of the United States of America, 109(6), 2015-2020.

Tanwar, M., Dada, T., \& Dada, R. (2010). Axenfeld-Rieger syndrome associated with congenital glaucoma and cytochrome P4501B1 gene mutations. Case Reports in Medicine, 2010.

Tumer, Z., \& Bach-Holm, D. (2009). Axenfeld-Rieger syndrome and spectrum of PITX2 and FOXC1 mutations. European Journal of Human Genetics, 17(12), 1527-1539.

Vithana, E. N., Khor, C. C., Qiao, C., Nongpiur, M. E., George, R., Chen, L. J., ... Aung, T. (2012). Genome-wide association analyses identify three new susceptibility loci for primary angle closure glaucoma. Nature Genetics, 44(10), 1142-1146.

Weisschuh, N., Wolf, C., Wissinger, B., \& Gramer, E. (2008). A novel mutation in the FOXC1 gene in a family with Axenfeld-Rieger syndrome and Peters' anomaly. Clinical Genetics, 74(5), 476-480.

World Health Organization, United Nations Children's Fund. 2009. WHO child growth standards and the identification of severe acute malnutrition in infants and children: A Joint Statement. World Health Organization.

\section{SUPPORTING INFORMATION}

Additional Supporting Information may be found online in the supporting information tab for this article.

How to cite this article: Carmona S, da Luz Freitas M, Froufe $\mathrm{H}$, et al. Novel de novo FOXC1 nonsense mutation in an Axenfeld-Rieger syndrome patient. Am J Med Genet Part A. 2017;173A:1607-1610. https://doi.org/10.1002/ajmg.a.38234 